\title{
ENERGETICS OF THE UNDAMPED STOCHASTIC HARMONIC OSCILLATOR*
}

\author{
Michą Mandrysz, BartŁomiej Dybiec \\ Marian Smoluchowski Institute of Physics \\ and Mark Kac Center for Complex Systems Research, Jagiellonian University \\ Łojasiewicza 11, 30-348 Kraków, Poland
}

(Received April 23, 2018)

The harmonic oscillator is one of fundamental models in physics. In stochastic thermodynamics, such models are usually accompanied with both stochastic and damping forces, acting as energy counter-terms. Here, on the other hand, we study properties of the undamped harmonic oscillator driven by additive noises. Consequently, the popular cases of Gaussian white noise, Markovian dichotomous noise and Ornstein-Uhlenbeck noise are analyzed from the energy point of view employing both analytical and numerical methods. In accordance to one's expectations, we confirm that energy is pumped into the system. We demonstrate that, as a function of time, initially total energy displays abrupt oscillatory changes, but then transits to the linear dependence in the long-time limit. Kinetic and potential parts of the energy are found to display oscillatory dependence at all times.

DOI:10.5506/APhysPolB.49.871

\section{Introduction}

Many situations in natural sciences can be successfully described in the stochastic manner [1-3]. In such an approach, it is usually assumed that complex interactions of the system at hand with its environment can be modeled with the use of noise. The choice of an appropriate noise-type is case-dependent. If individual collisions are approximated as bounded and independent, the noise is assumed to be the Gaussian white noise. In more general and more realistic scenarios, the noise does not need to be white nor Gaussian [4].

* Presented at the XXX Marian Smoluchowski Symposium on Statistical Physics, Kraków, Poland, September 3-8, 2017. 
Indeed, such effective approaches turn out to be very useful. There is a growing interest in examination of fluctuations and their role in stochastic systems with the special attention to noise induced effects, such as stochastic resonance [5, 6], resonant activation [7], synchronization [8] and direct transport (ratcheting effect) [9] being just a few examples. Moreover, thermodynamical properties of stochastic systems such as energetics [10] and fluctuation theorems [11] became important part of research in the field of stochastic thermodynamics [12] both at the theoretical [13] and the experimental levels [14, 15].

Here, we study analytically and numerically energetic properties of the undamped stochastic harmonic oscillator with special attention to time dependence of energies (total, kinetic and potential). The studied setup, required theory and main results are presented in Model and results (Sec. 2). The paper is closed with Summary and discussion (Sec. 3).

\section{Model and results}

In order to present the studied model in a broader context, we start our presentation from the damped (stochastic) harmonic oscillator [16, 17]. We use the convention employed in [18, 19]. In such dimensional units [18, 19], the evolution of the state variable $x(t)$ is described by the Langevin equation

$$
m \frac{\mathrm{d}^{2} x(t)}{\mathrm{d} t^{2}}=-\gamma m \frac{\mathrm{d} x(t)}{\mathrm{d} t}-k x(t)+\sqrt{2 \gamma k_{\mathrm{B}} T m} \xi(t),
$$

where $x(t)$ represents position, $m$ particle mass, $T$ temperature, $k_{\mathrm{B}}$ Boltzmann constant and $\gamma$ the damping coefficient. The form of Eq. (1) assures that the stochastic harmonic oscillator fulfills the equipartition theorem $[18,19]$. At this stage, $\xi(t)$ in Eq. (1) stands for the Gaussian white noise satisfying

$$
\langle\xi(t)\rangle=0
$$

and

$$
\langle\xi(t) \xi(s)\rangle=\delta(t-s) .
$$

The Gaussian white noise is interpreted as an interaction term between the harmonic oscillator and the thermal bath of temperature T. Equation (1) is the Newton second law accounting for such a random force. Due to the random force, both deterministic variables position $x(t)$ and velocity $v(t)=\dot{x}(t)$ become random variables distributed according to some probability density $P(x, v ; t)$. Evolution of the probability distribution of finding the system in a state characterized by $(x(t), v(t))$ is described $[18,19]$ by the diffusion 
(Fokker-Planck) equation

$$
\frac{\partial P(x, v ; t)}{\partial t}=\left[-v \frac{\partial}{\partial x}+\frac{\partial}{\partial v}\left(\gamma v+\frac{V^{\prime}(x)}{m}\right)+\gamma \frac{k_{\mathrm{B}} T}{m} \frac{\partial^{2}}{\partial v^{2}}\right] P(x, v ; t),
$$

where $V(x)=\frac{k}{2} x^{2}$ and $V^{\prime}(x)=k x$. In the damped case, for any potential $V(x)$ such that $V(x) \rightarrow \infty$ when $x \rightarrow \pm \infty$, Eq. (4) has the stationary solution of the Boltzmann-Gibbs-type

$$
P(x, v) \propto \exp \left[-\frac{1}{k_{\mathrm{B}} T}\left(\frac{m v^{2}}{2}+V(x)\right)\right] .
$$

The exponent in Eq. (5) is the total energy $\mathcal{E}$ which is the sum of kinetic $\mathcal{E}_{\mathrm{k}}$ and potential $\mathcal{E}_{\mathrm{p}}$ energies. Since the energy of the system $\mathcal{E}=\mathcal{E}_{\mathrm{k}}+\mathcal{E}_{\mathrm{p}}=$ $\frac{1}{2} m v^{2}+\frac{k}{2} x^{2}$ depends on its state $(x(t), v(t))$, the energies as such also become random variables.

Equation (1) can be rewritten as a set of two first order equations

$$
\left\{\begin{array}{l}
\frac{\mathrm{d} x(t)}{\mathrm{d} t}=v(t), \\
\frac{\mathrm{d} v(t)}{\mathrm{d} t}=-\gamma v(t)-\omega^{2} x(t)+\sqrt{\frac{2 \gamma k_{\mathrm{B}} T}{m}} \xi(t),
\end{array}\right.
$$

where $\omega^{2}=k / m$. For the parabolic potential, these equations are linear, consequently standard methods of solving linear differential equations can be applied [19, 20]. The system described by Eq. (1) or Eq. (6) in the presence of simple noises, e.g. the Gaussian white noise, the dichotomous noise or the Ornstein-Uhlenbeck noise, can be studied analytically [19, 20].

From Eq. (6), one can derive equations for moments $\left\langle v^{2}(t)\right\rangle$ and $\left\langle x^{2}(t)\right\rangle$ from which evolution of energies can be calculated [12]. Multiplying the relation

$$
\frac{\mathrm{d} x(t)}{\mathrm{d} t}=v(t)
$$

by $k x(t)$ and performing (ensemble) averaging $\langle\ldots\rangle$ results in

$$
\frac{\mathrm{d}}{\mathrm{d} t}\left\langle\mathcal{E}_{\mathrm{p}}(t)\right\rangle=k\langle v(t) x(t)\rangle .
$$

Multiplying the second line of Eq. (6) by $v(t)$ and (ensemble) averaging $\langle\ldots\rangle$ gives rise to

$$
\frac{\mathrm{d}}{\mathrm{d} t}\left\langle\mathcal{E}_{\mathrm{k}}(t)\right\rangle=-\gamma m\left\langle v^{2}(t)\right\rangle-k\langle v(t) x(t)\rangle+\sqrt{2 \gamma k_{\mathrm{B}} T m}\langle\xi(t) v(t)\rangle .
$$

The addition of Eqs. (8) and (9) produces the formula for the time derivative of the average total energy $\langle\mathcal{E}\rangle$

$$
\frac{\mathrm{d}}{\mathrm{d} t}\langle\mathcal{E}(t)\rangle=-\gamma m\left\langle v^{2}(t)\right\rangle+\sqrt{2 \gamma k_{\mathrm{B}} T m}\langle\xi(t) v(t)\rangle .
$$


The various modifications of the system described by Eq. (1) have been studied [21-26]. Here, we study properties of a simpler system. Starting from now, we focus on the undamped stochastic harmonic oscillator [27], i.e. we assume that in Eq. (1) there is no damping term $(-\gamma m v(t))$. Consequently, we study the time dependence of the energy in the stochastic harmonic oscillator described by the following Langevin equation:

$$
\frac{\mathrm{d}^{2} x(t)}{\mathrm{d} t^{2}}=-\omega^{2} x(t)+\sqrt{h} \xi(t)
$$

or equivalently by

$$
\frac{\mathrm{d} v(t)}{\mathrm{d} t}=-\omega^{2} x(t)+\sqrt{h} \xi(t),
$$

where $h$ is the parameter scaling the noise, $v(t)=\frac{\mathrm{d} x(t)}{\mathrm{d} t}$ and $\omega^{2}=k / m$. Due to absence of the damping term, $h$ becomes an independent of $\gamma$ parameter scaling the noise. In the simplest case of the Gaussian white noise, the analytical evaluation of formulas (8)-(10) with the initial condition $x(0)=0$ and $v(0)=0$, followed by the integration, yields

$$
\begin{aligned}
\left\langle\mathcal{E}_{\mathrm{k}}(t)\right\rangle & =h \frac{2 \omega t+\sin (2 \omega t)}{8 \omega}, \\
\left\langle\mathcal{E}_{\mathrm{p}}(t)\right\rangle & =h \frac{2 \omega t-\sin (2 \omega t)}{8 \omega}, \\
\langle\mathcal{E}(t)\rangle & =h \frac{t}{2} .
\end{aligned}
$$

In other words, in the case of missing damping (dissipative) term in Eq. (1), the Gaussian white noise effectively pumps energy into the system. Moreover, one easily notices that for sufficiently large $t$, approximately half of the total energy is stored into kinetic one, while the remaining half is stored as the potential energy. With increasing $t$, the quality of this approximation improves and the approximation becomes exact as $t \rightarrow \infty$.

Now, we turn our attention to the case of Markovian symmetric dichotomous noise $\xi_{\mathrm{DN}}(t)$ replacing the Gaussian white noise, see [1]. We consider Markovian symmetric dichotomous noise taking values $\pm 1\left(\xi_{\mathrm{DN}}(t) \in\right.$ $\{-1,1\})$ obeying

$$
\left\langle\xi_{\mathrm{DN}}(t)\right\rangle=0
$$

and

$$
\left\langle\xi_{\mathrm{DN}}(t) \xi_{\mathrm{DN}}(s)\right\rangle=\exp [-2 \lambda|t-s|],
$$

where $\lambda$ is the transition rate between states. Additionally, we have assumed that

$$
\xi_{\mathrm{DN}}(0) \in\{-1,+1\}
$$


with probability $1 / 2$. Exact formulas for kinetic, potential and total energies read

$$
\begin{aligned}
\left\langle\mathcal{E}_{\mathrm{k}}(t)\right\rangle & =h \frac{\left(4 \lambda^{2}+\omega^{2}\right) \lambda t-4 \lambda^{2}+e^{-2 \lambda t}\left(4 \lambda^{2} \cos (\omega t)-2 \lambda \omega \sin (\omega t)\right)}{\left(4 \lambda^{2}+\omega^{2}\right)^{2}}+\Delta(t), \\
\left\langle\mathcal{E}_{\mathrm{p}}(t)\right\rangle & =h \frac{\left(4 \lambda^{2}+\omega^{2}\right) \lambda t+\omega^{2}-e^{-2 \lambda t}\left(\omega^{2} \cos (\omega t)-2 \lambda \omega \sin (\omega t)\right)}{\left(4 \lambda^{2}+\omega^{2}\right)^{2}}-\Delta(t), \\
\langle\mathcal{E}(t)\rangle & =\left\langle\mathcal{E}_{\mathrm{k}}(t)\right\rangle+\left\langle\mathcal{E}_{\mathrm{p}}(t)\right\rangle,
\end{aligned}
$$

where $\Delta(t)$ is given by

$$
\Delta(t)=h \frac{\lambda \sin (2 \omega t)+\omega \sin ^{2}(\omega t)}{8 \lambda^{2} \omega+2 \omega^{3}} .
$$

In the limit of $t \rightarrow \infty$, the average total energy is increasing linearly with time with the coefficient $\frac{2 \lambda h}{4 \lambda^{2}+\omega^{2}}$, i.e. asymptotically

$$
\langle\mathcal{E}(t)\rangle \approx \frac{2 \lambda h}{4 \lambda^{2}+\omega^{2}} t
$$

while the kinetic and potential parts of the total energy have the asymptotic form

$$
\left\langle\mathcal{E}_{\mathrm{k}}(t)\right\rangle \approx \frac{\lambda h}{4 \lambda^{2}+\omega^{2}} t \simeq \frac{1}{2}\langle\mathcal{E}(t)\rangle
$$

and

$$
\left\langle\mathcal{E}_{\mathrm{p}}(t)\right\rangle \approx \frac{\lambda h}{4 \lambda^{2}+\omega^{2}} t \simeq \frac{1}{2}\langle\mathcal{E}(t)\rangle .
$$

Therefore, for the Markovian symmetric dichotomous noise, analogously like for the Gaussian white noise, asymptotically energy is equidistributed among kinetic and potential energies, i.e. half of the energy is stored in the form of kinetic (potential) energy.

Finally, we have replaced the Gaussian white noise with the OrnsteinUhlenbeck process [28] which is described by the following Langevin equation

$$
\frac{\mathrm{d} \xi_{\mathrm{OU}}(t)}{\mathrm{d} t}=-\rho \xi_{\mathrm{OU}}(t)+\xi(t)
$$

where $\xi(t)$ is the Gaussian white noise, see Eqs. (2) and (3). For such a choice, the Ornstein-Uhlenbeck process fulfills

$$
\left\langle\xi_{\mathrm{OU}}(t)\right\rangle=0
$$


and

$$
\left\langle\xi_{\mathrm{OU}}(t) \xi_{\mathrm{OU}}(s)\right\rangle=\frac{1}{2 \rho} \exp [-\rho|t-s|],
$$

with the additional assumption that $\xi_{\mathrm{OU}}(-\infty)=0$. Due to its similar form, results for the Ornstein-Uhlenbeck noise closely resemble former equations for the Markovian symmetric dichotomous noise

$$
\begin{aligned}
\left\langle\mathcal{E}_{\mathrm{k}}(t)\right\rangle & =h \frac{\left(\rho^{2}+\omega^{2}\right) 2 \rho t-3 \rho^{2}+\omega^{2}+e^{-\rho t}\left(4 \rho^{2} \cos (\omega t)-4 \rho \omega \sin (\omega t)\right)}{8 \rho\left(\rho^{2}+\omega^{2}\right)^{2}}+\tilde{\Delta}(t), \\
\left\langle\mathcal{E}_{\mathrm{p}}(t)\right\rangle & =h \frac{\left(\rho^{2}+\omega^{2}\right) 2 \rho t+3 \omega^{2}-\rho^{2}-e^{-\rho t}\left(4 \omega^{2} \cos (\omega t)+4 \rho \omega \sin (\omega t)\right)}{8 \rho\left(\rho^{2}+\omega^{2}\right)^{2}}-\tilde{\Delta}(t), \\
\langle\mathcal{E}(t)\rangle & =\left\langle\mathcal{E}_{\mathrm{k}}(t)\right\rangle+\left\langle\mathcal{E}_{\mathrm{p}}(t)\right\rangle,
\end{aligned}
$$

where $\tilde{\Delta}(t)$ reads

$$
\tilde{\Delta}(t)=h \frac{\rho \sin (2 \omega t)-\omega \cos (2 \omega t)}{8 \rho^{3} \omega+8 \rho \omega^{3}} .
$$

In the limit of $t \rightarrow \infty$, the average total energy also grows linearly with time, but with the coefficient $\frac{2 \lambda h}{4 \lambda^{2}+\omega^{2}}$, i.e. asymptotically

$$
\langle\mathcal{E}(t)\rangle \approx \frac{h}{2\left(\rho^{2}+\omega^{2}\right)} t .
$$

For the kinetic and potential energies, we obtain

$$
\left\langle\mathcal{E}_{\mathrm{k}}(t)\right\rangle \approx \frac{h}{4\left(\rho^{2}+\omega^{2}\right)} t \simeq \frac{1}{2}\langle\mathcal{E}(t)\rangle
$$

and

$$
\left\langle\mathcal{E}_{\mathrm{p}}(t)\right\rangle \approx \frac{h}{4\left(\rho^{2}+\omega^{2}\right)} t \simeq \frac{1}{2}\langle\mathcal{E}(t)\rangle .
$$

Here, again, energy is asymptotically equidistributed between kinetic and potential form. The last case, also studied numerically, corresponds to the combined independent action of all studied noises, i.e. it is a sum of all studied noises.

Numerical results for time dependence of average total $\langle\mathcal{E}(t)\rangle$, potential $\left\langle\mathcal{E}_{\mathrm{p}}(t)\right\rangle$ and kinetic $\left\langle\mathcal{E}_{\mathrm{k}}(t)\right\rangle$ energies for the undamped stochastic harmonic oscillator are presented in Figs. 1-4. Subsequent figures correspond to different driving noise: the Gaussian white, dichotomous $(\lambda=1)$, OrnsteinUhlenbeck $(\rho=1)$, and the combined action of all these noises. Analytical solutions have been constructed with the help of Eqs. (8)-(10) and (analytical) solution of Eq. (11). 


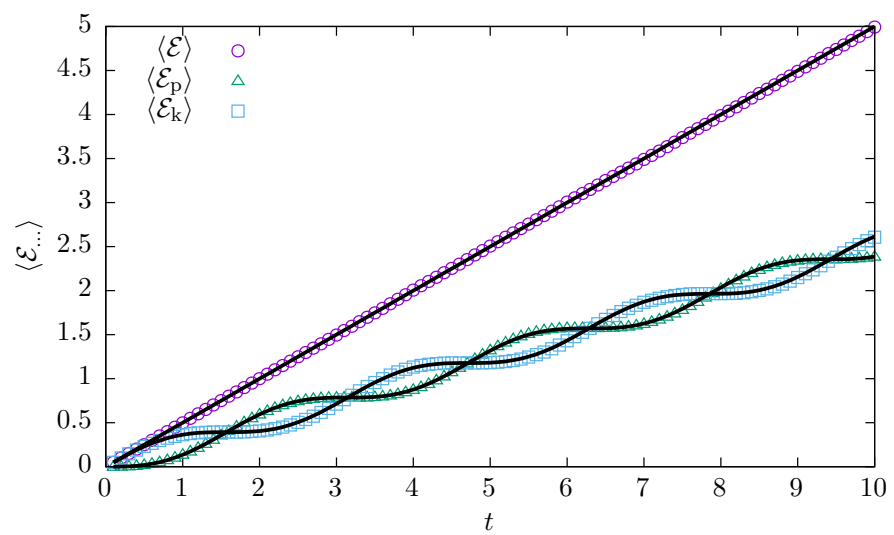

Fig. 1. Average total $(\langle\mathcal{E}(t)\rangle)$, kinetic $\left(\left\langle\mathcal{E}_{\mathrm{k}}(t)\right\rangle\right)$ and potential $\left(\left\langle\mathcal{E}_{\mathrm{p}}(t)\right\rangle\right)$ energies for the undamped stochastic harmonic oscillator driven by the Gaussian white noise. Solid lines present theoretical values given by Eqs. (13)-(15).

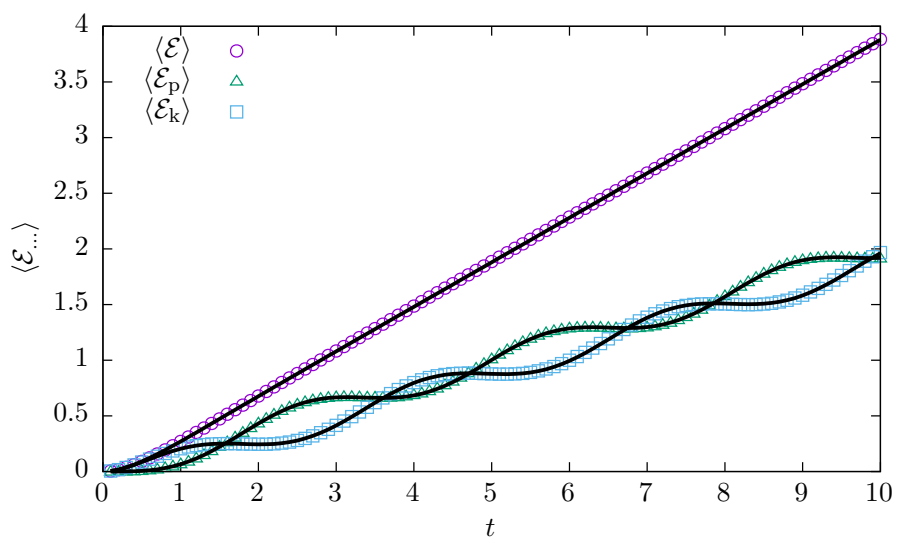

Fig. 2. The same as in Fig. 1 for the Markovian symmetric dichotomous noise with $\lambda=1$. Solid lines present theoretical values given by Eqs. (19)-(21).

Numerical (Monte Carlo) results for the (undamped) stochastic harmonic oscillator have been constructed by the algorithm presented in [29]. For the sake of simplicity, it was assumed that $h=1, x(0)=0$ and $v(0)=0$. As it can be seen from Figs. 1-3 for the parabolic potential, the theoretical curves nicely corroborate numerical simulations. To further compare analytical results with numerical approximations, we have repeated simulations for the Markovian dichotomous noise with longer correlation time, which corresponds to a smaller switching rate $\lambda=1 / 10$, see Fig. 5. Analogously, the Ornstein-Uhlenbeck noise with a smaller damping $\rho=1 / 10$ has been studied, see Fig. 6 . In both cases, numerical simulations with longer correla- 
tion times are in perfect accordance with the analytical results. For nonzero initial conditions, the complete agreement between numerical simulations and analytical formulas has been also recorded (results not shown).

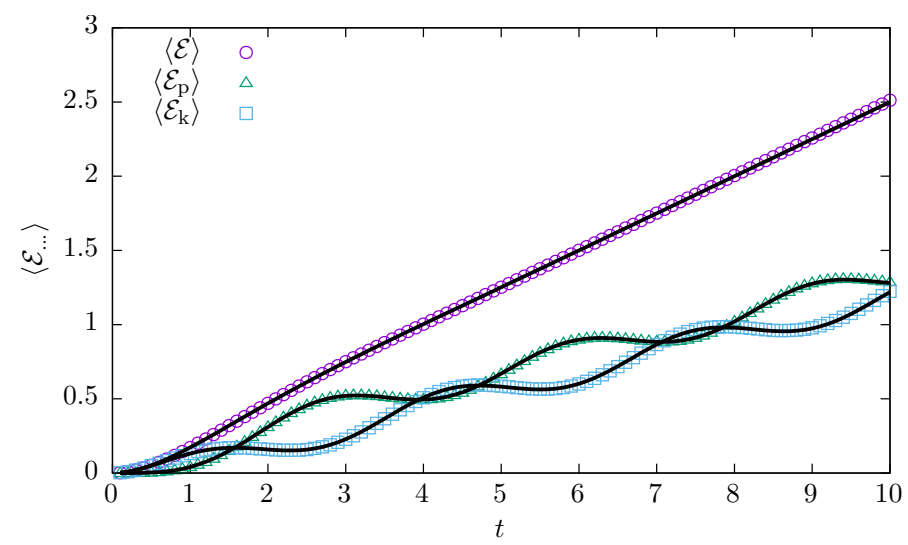

Fig. 3. The same as in Fig. 1 for the Ornstein-Uhlenbeck noise with $\rho=1$. Solid lines present theoretical values given by Eqs. (29)-(31).

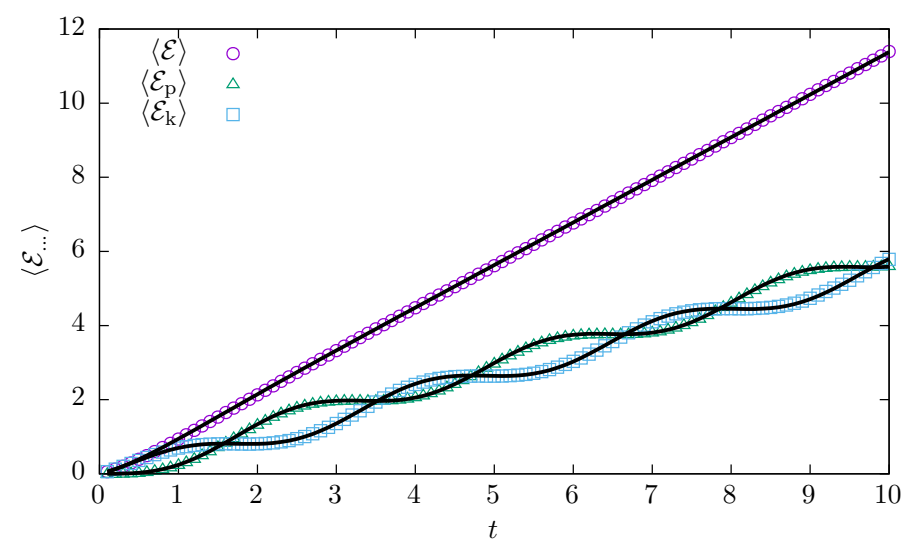

Fig. 4. The same as in Fig. 1 for the sum of all considered noises. Solid lines present theoretical values given by sums of appropriate energies given by Eqs. (13)-(15), (19)-(21) and (29)-(31).

Noise in Eq. (11) is additive. Therefore, combined action of various types of independent noises also results in pumping of the energy into the system. The injected energy is the sum of pumped energies for each noise independently. Figure 4 presents results for the sum of the Gaussian white noise, the Markovian dichotomous noise and the Ornstein-Uhlenbeck noise. The effect of combined action of all considered noises is the direct consequence of their independence, see Eqs. (8)-(10) and Eq. (11). 

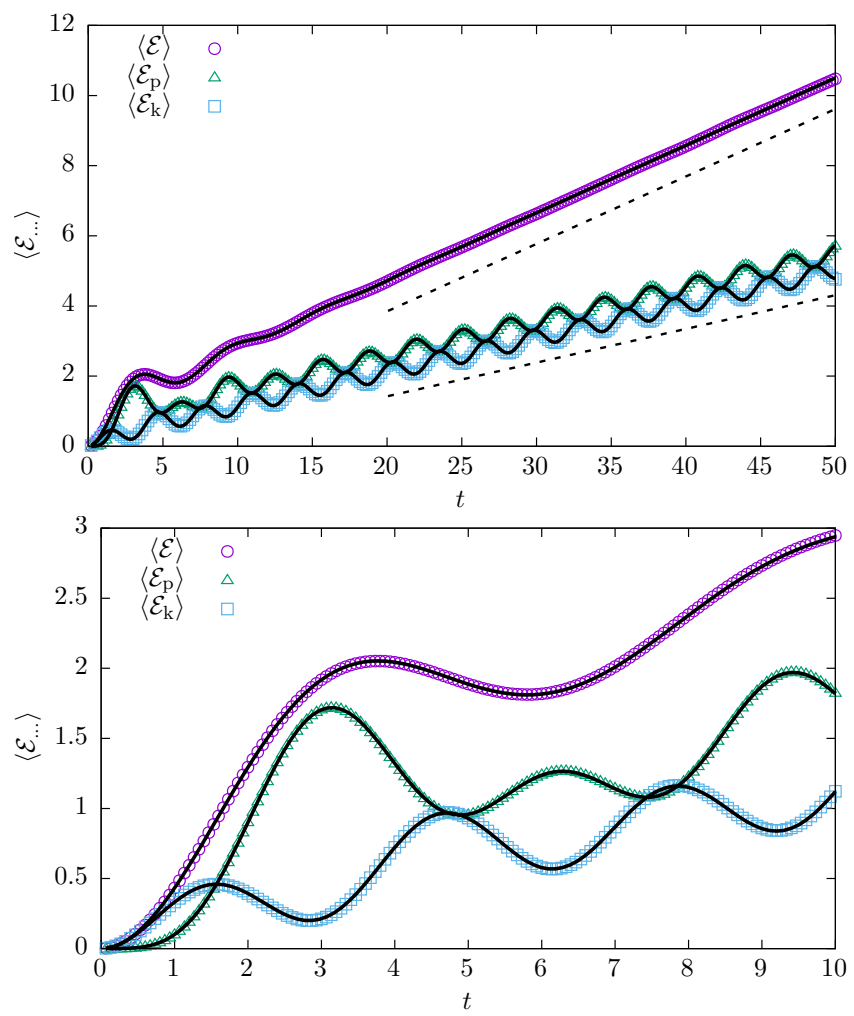

Fig. 5. The same as in Fig. 1 for the Markovian symmetric dichotomous noise with $\lambda=1 / 10$. Bottom panel presents short-time dependence. Solid lines present theoretical values given by Eqs. (19)-(21). Dashed lines in the top panel present asymptotic scaling given by Eqs. (23)-(25).

Figures 5 and 6 demonstrate not only the short-time dependence of energies (bottom panel) but also longer time dependence (top panel). After the subtraction of the linear trend and after the transient period, potential and kinetic energies oscillate periodically. Such an effect is an inherent property of the harmonic oscillator because its period does not depend on its energy, see [30]. Therefore, despite pumping of the energy into the system, its period remains unchanged. Consequently, clear oscillations of potential and kinetic energies which are superimposed on the linear trend are visible, see Eqs. (13)-(15), (19)-(21) and (29)-(31) and Figs. 5-6. 

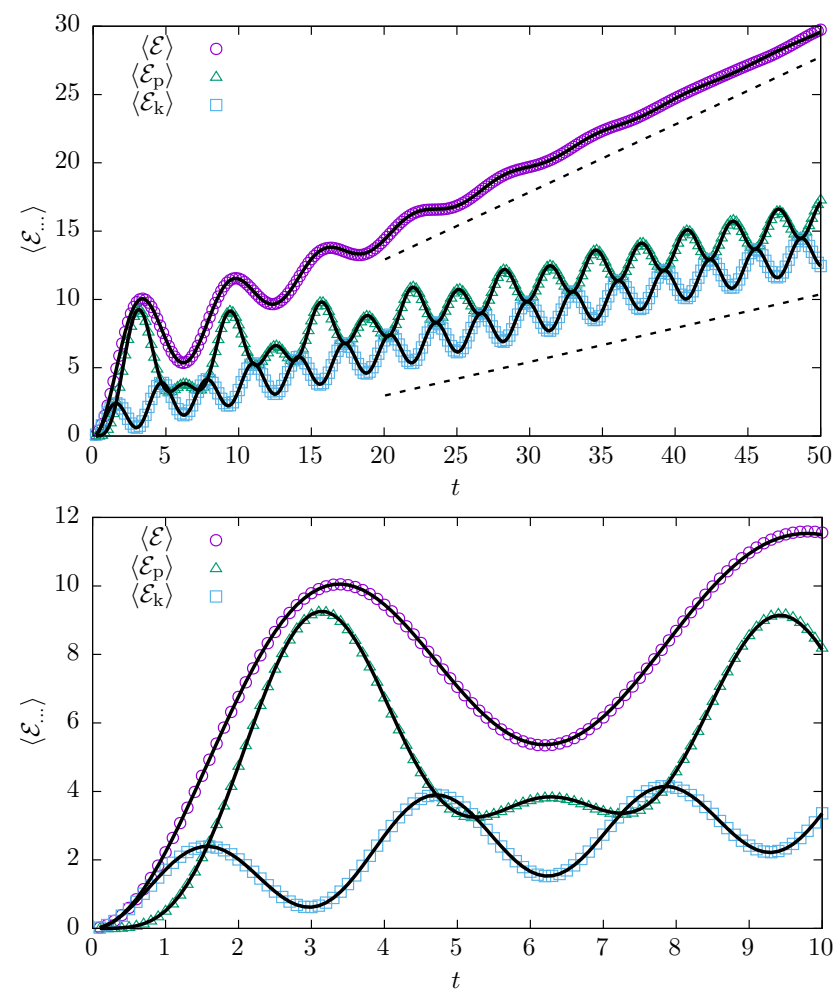

Fig. 6. The same as in Fig. 1 for the Ornstein-Uhlenbeck noise with $\rho=1 / 10$. Bottom panel presents short-time dependence. Solid lines present theoretical values given by Eqs. (29)-(31). Dashed lines in the top panel present asymptotic scaling given by Eqs. (33)-(35).

\section{Summary and conclusions}

Using analytical calculations and computer simulations, we have studied the time evolution of energy (total, kinetic and potential) in the undamped stochastic harmonic oscillator. Due to lack of damping, the energy balance is violated and, consequently, the energy is pumped into the system. In the simplest cases of the Gaussian white noise, the Markovian dichotomous noise and the Ornstein-Uhlenbeck noise, we have derived formulas for the time dependence of the total, potential and kinetic energies. These formulas perfectly fit results of Monte Carlo simulations. In the long-time limit energies (total, kinetic and potential) of the undamped stochastic harmonic oscillator grow linearly in time. The linear growth is decorated by superimposed oscillations which are well-visible for kinetic and potential energies at all times. The observed oscillations in the total energy, quickly disappear due to half-cycle phase shifts in the time dependence of kinetic and potential energies. 
This project was supported by the National Science Centre, Poland (NCN) grant (2014/13/B/ST2/02014). Discussions with Karol Capała are greatly acknowledged. Computer simulations have been performed at the Academic Computer Center Cyfronet, AGH University of Science and Technology (Kraków, Poland) under CPU grant DynStoch.

\section{REFERENCES}

[1] W. Horsthemke, R. Lefever, Noise-inducted Transitions. Theory and Applications in Physics, Chemistry, and Biology, Springer Verlag, Berlin 1984.

[2] P. Hänggi, P. Talkner, M. Borkovec, Rev. Mod. Phys. 62, 251 (1990).

[3] L. Gammaitoni, P. Hänggi, P. Jung, F. Marchesoni, Eur. Phys. J. B 69, 1 (2009).

[4] G. Samorodnitsky, M.S. Taqqu, Stable non-Gaussian Random Processes: Stochastic Models with Infinite Variance, Chapman and Hall, New York 1994.

[5] L. Gammaitoni, P. Hänggi, P. Jung, F. Marchesoni, Rev. Mod. Phys. 70, 223 (1998).

[6] V.S. Anishchenko, A.B. Neiman, F. Moss, L. Schimansky-Geier, Sov. Phys. Usp. 42, 7 (1999).

[7] C.R. Doering, J.C. Gadoua, Phys. Rev. Lett. 69, 2318 (1992).

[8] V.S. Anishchenko, A.B. Neiman, in Stochastic Dynamics, (ed.)

L. Schimansky-Geier, T. Pöshel, Springer Verlag, Berlin 1997, pp. 154-166.

[9] P. Reimann, Phys. Rep. 361, 57 (2002).

[10] K. Sekimoto, Stochastic Energetics, Springer Verlag, Berlin 2010.

[11] U. Seifert, Rep. Prog. Phys. 75, 126001 (2012).

[12] T. Tomé, M.J. De Oliveira, Stochastic Dynamics and Irreversibility, Springer Verlag, Berlin 2016.

[13] R.L. Stratonovich, Nonlinear Nonequilibrium Thermodynamics I: Linear and Nonlinear Fluctuation-Dissipation Theorems, Springer Verlag, Berlin 1992.

[14] D. Collin et al., Nature 437, 231 (2005).

[15] S. Schuler et al., Phys. Rev. Lett. 94, 180602 (2005).

[16] M. Gitterman, The Noisy Oscillator: The First Hundred Years, from Einstein Until Now, World Scientific, Singapore 2005.

[17] M. Gitterman, The Noisy Oscillator: Random Mass, Frequency, Damping, World Scientific, Singapore 2013.

[18] R. Kubo, Rep. Prog. Phys. 29, 255 (1966).

[19] H. Risken, The Fokker-Planck Equation. Methods of Solution and Application, Springer Verlag, Berlin 1984.

[20] X. Mao, Stochastic Differential Equations and Applications, Woodhead Publishing, Oxford 2007. 
[21] M. Gitterman, Phys. Rev. E 69, 041101 (2004).

[22] V. Méndez, W. Horsthemke, P. Mestres, D. Campos, Phys. Rev. E 84, 041137 (2011).

[23] M. Gitterman, D.A. Kessler, Phys. Rev. E 87, 022137 (2013).

[24] M. Gitterman, J. Stat. Phys. 157, 376 (2014).

[25] S. Joubaud, N. Garnier, S. Ciliberto, J. Stat. Mech. 2007, P09018 (2007).

[26] M. Yaghoubi, M.E. Foulaadvand, A. Bérut, J. Łuczka, J. Stat. Mech. 2017, 113206 (2017).

[27] N. Lin, S. Lototsky, Commun. Stoch. Anal. 5, 13 (2011).

[28] C.W. Gardiner, Handbook of Stochastic Methods for Physics, Chemistry and Natural Sciences, Springer Verlag, Berlin 2009.

[29] A.H. Melbø, D.J. Higham, Appl. Numer. Math. 51, 89 (2004).

[30] L.D. Landau, E.M. Lifshits, Theoretical Physics: Mechanics, Pergamon Press, Oxford 1988. 